Kragujevac Journal of Mathematics

Volume 42(4) (2018), Pages 485-493.

\title{
WHEN IS A BI-JORDAN HOMOMORPHISM BI-HOMOMORPHISM?
}

\author{
A. ZIVARI-KAZEMPOUR ${ }^{1}$
}

\begin{abstract}
For Banach algebras $\mathcal{A}$ and $\mathcal{B}$, we show that if $\mathcal{U}=\mathcal{A} \times \mathcal{B}$ is commutative (weakly commutative), then each bi-Jordan homomorphism from $\mathcal{U}$ into a semisimple commutative Banach algebra $\mathcal{D}$ is a bi-homomorphism. We also prove the same result for 3-bi-Jordan homomorphism with the additional hypothesis that the Banach algebra $\mathcal{U}$ is unital.
\end{abstract}

\section{IntRoduction}

Let $\mathcal{A}$ and $\mathcal{B}$ be complex Banach algebras and $\varphi: \mathcal{A} \rightarrow \mathcal{B}$ be a linear map. Then $\varphi$ is called an n-homomorphism if for all $a_{1}, a_{2}, \ldots a_{n} \in \mathcal{A}$,

$$
\varphi\left(a_{1} a_{2} \ldots a_{n}\right)=\varphi\left(a_{1}\right) \varphi\left(a_{2}\right) \ldots \varphi\left(a_{n}\right) .
$$

The concept of an n-homomorphism was studied for complex algebras by Hejazian et al. in [5]. A 2-homomorphism is then just a homomorphism, in the usual sense. One may refer to [1] for certain properties of 3-homomorphisms.

The notion of n-Jordan homomorphisms was dealt with firstly by Herstein in [6]. A linear map $\varphi$ between Banach algebras $\mathcal{A}$ and $\mathcal{B}$ is called an n-Jordan homomorphism if

$$
\varphi\left(a^{n}\right)=\varphi(a)^{n}, \quad a \in \mathcal{A} .
$$

A 2-Jordan homomorphism is called simply a Jordan homomorphism.

It is obvious that each n-homomorphism is an n-Jordan homomorphism, but in general the converse is false. The converse statement may be true under certain conditions. For example, it is shown in [2] that every n-Jordan homomorphism between two commutative Banach algebras is an n-homomorphism for $n \in\{2,3,4\}$,

Key words and phrases. n-bi-homomorphism, n-bi-Jordan homomorphism, weakly commutative. 2010 Mathematics Subject Classification. Primary: 47B48. Secondary: 46L05, 46H25.

Received: March 03, 2017.

Accepted: April 04, 2017. 
and this result extended to the case $n=5$ in [3]. Lee in [7] generalized this result and proved it for all $n \in \mathbb{N}$. See also [4] for another proof of Lee's theorem.

Zelazko in [9] has given a characterization of Jordan homomorphism, that we mention in the following (see also [8]). We refer to [10] for another approach to the same result.

Theorem 1.1. Suppose that $\mathcal{A}$ is a Banach algebra, which need not be commutative, and suppose that $\mathcal{B}$ is a semisimple commutative Banach algebra. Then each Jordan homomorphism $\varphi: \mathcal{A} \rightarrow \mathcal{B}$ is a homomorphism.

Also it is shown in [11] that Theorem 1.1 is valid for 3-Jordan homomorphism with the extra condition that the Banach algebra $\mathcal{A}$ is unital. Some significant results concerning Jordan homomorphisms and their generalizations on Banach algebras obtained by the author in [12].

Throughout the paper, let $\mathcal{U}=\mathcal{A} \times \mathcal{B}$. Then $\mathcal{U}$ is a Banach algebra for the multiplication

$$
(a, b)(x, y)=(a x, b y), \quad(a, b),(x, y) \in \mathcal{U}
$$

and with norm

$$
\|(a, b)\|=\|a\|+\|b\| .
$$

Let $\mathcal{D}$ be a complex Banach algebra. A bilinear map is a function $\varphi: \mathcal{U} \rightarrow \mathcal{D}$ such that for any $a \in \mathcal{A}$ the map $b \mapsto \varphi(a, b)$ is linear map from $\mathcal{B}$ to $\mathcal{D}$, and for any $b \in \mathcal{B}$ the map $a \mapsto \varphi(a, b)$ is linear map from $\mathcal{A}$ to $\mathcal{D}$.

A bilinear map $\varphi$ is called an n-bi-homomorphism if for all $\left(a_{i}, b_{i}\right) \in \mathcal{U}$,

$$
\varphi\left(a_{1} a_{2} \ldots a_{n}, b_{1} b_{2} \ldots b_{n}\right)=\varphi\left(a_{1}, b_{1}\right) \varphi\left(a_{2}, b_{2}\right) \ldots \varphi\left(a_{n}, b_{n}\right),
$$

and it is called an n-bi-Jordan homomorphism if

$$
\varphi\left(a^{n}, b^{n}\right)=\varphi(a, b)^{n}, \quad(a, b) \in \mathcal{U} .
$$

The concept of an n-bi-Jordan homomorphism introduced by the author in [13]. A (2-bi-Jordan) 2-bi-homomorphism is called simply a (bi-Jordan) bi-homomorphism.

It is obvious that each n-bi-homomorphism is n-bi-Jordan homomorphism, but in general the converse is not true.

Recently, the author proved [13] that every bi-Jordan homomorphism from unital commutative Banach algebra $\mathcal{U}$ into a semisimple commutative Banach algebra $\mathcal{D}$ is a bi-homomorphism.

In this paper, we extended this result for nonunital Banach algebra $\mathcal{U}$. We also prove the same result for 3-bi-Jordan homomorphism with the additional hypothesis that the Banach algebra $\mathcal{U}$ is unital.

\section{Characterization of Bi-Jordan Homomorhisms}

The following Theorem is the generalization of Theorem 4 of [13]. 
Theorem 2.1. Every bi-Jordan homomorphism $\varphi$ from commutative Banach algebra $\mathcal{U}$ into a semisimple commutative Banach algebra $\mathcal{D}$ is a bi-homomorphism.

Proof. We first assume that $\mathcal{D}=\mathbb{C}$ and let $\varphi: \mathcal{U} \rightarrow \mathbb{C}$ be a bi-Jordan homomorphism. Then for all $(a, b) \in \mathcal{U}$,

$$
\varphi\left(a^{2}, b^{2}\right)=\varphi(a, b)^{2} .
$$

Replacing $a$ by $a+x$ and $b$ by $b+y$ in (2.1), gives

$$
\varphi\left(a^{2}+x^{2}+2 a x, b^{2}+y^{2}+2 b y\right)=\varphi(a+x, b+y)^{2} .
$$

By Lemma 1 of [13], for all $(a, b),(x, y) \in \mathcal{U}$ we have

$$
\varphi\left(a^{2}, b y\right)=\varphi(a, b) \varphi(a, y) \quad \text { and } \quad \varphi\left(a x, b^{2}\right)=\varphi(a, b) \varphi(x, b) .
$$

It follows from (2.2) and (2.3) that

$$
2 \varphi(a x, b y)=\varphi(a, b) \varphi(x, y)+\varphi(a, y) \varphi(x, b),
$$

for all $(a, b),(x, y) \in \mathcal{U}$. Take $I=\varphi(a, b) \varphi(x, y), J=\varphi(a, y) \varphi(x, b)$ and $t=I-J$. Then we get

$$
t^{2}=I^{2}+J^{2}-2 I J, \quad 4 \varphi(a x, b y)^{2}=I^{2}+J^{2}+2 I J .
$$

By (2.4) and (2.5), we deduce

$$
\begin{aligned}
4 \varphi(a x, b y)^{2}+t^{2} & =2\left(I^{2}+J^{2}\right) \\
& =2\left[\varphi(a, b)^{2} \varphi(x, y)^{2}+\varphi(a, y)^{2} \varphi(x, b)^{2}\right] \\
& =2\left[\varphi\left(a^{2}, b^{2}\right) \varphi\left(x^{2}, y^{2}\right)+\varphi\left(a^{2}, y^{2}\right) \varphi\left(x^{2}, b^{2}\right)\right] \\
& =4 \varphi\left(a^{2} x^{2}, b^{2} y^{2}\right) \\
& =4 \varphi(a x, b y)^{2} .
\end{aligned}
$$

Hence, $t=0$, which proves that $I=J$. Thus, by (2.4) we have

$$
\varphi(a x, b y)=\varphi(a, b) \varphi(x, y),
$$

for all $(a, b),(x, y) \in \mathcal{U}$, so $\varphi$ is a bi-homomorphism.

Now suppose that $\mathcal{D}$ is semisimple and commutative. Let $\mathfrak{M}(\mathcal{D})$ be the maximal ideal space of $\mathcal{D}$. We associate with each $f \in \mathfrak{M}(\mathcal{D})$ a function $\varphi_{f}: \mathcal{U} \rightarrow \mathbb{C}$ defined by

$$
\varphi_{f}(a, b):=f(\varphi(a, b)), \quad(a, b) \in \mathcal{U} .
$$

Pick $f \in \mathfrak{M}(\mathcal{D})$ arbitrary. It is easy to see that $\varphi_{f}$ is a bi-Jordan homomorphism, so by the above argument it is a bi-homomorphism. Thus, by the definition of $\varphi_{f}$ we have

$$
f(\varphi(a x, b y))=f(\varphi(a, b)) f(\varphi(x, y))=f(\varphi(a, b) \varphi(x, y)) .
$$

Since $f \in \mathfrak{M}(\mathcal{D})$ was arbitrary and $\mathcal{D}$ is assumed to be semisimple,

$$
\varphi(a x, b y)=\varphi(a, b) \varphi(x, y),
$$

for all $(a, b),(x, y) \in \mathcal{U}$. This complete the proof. 
A bilinear map $\varphi: \mathcal{U} \rightarrow \mathcal{D}$ is called co-bi-homomorphism if

$$
\varphi(a x, b y)=-\varphi(a, b) \varphi(x, y),
$$

for all $(a, b),(x, y) \in \mathcal{U}$, and it is called co-bi-Jordan homomorphism if

$$
\varphi\left(a^{2}, b^{2}\right)=-\varphi(a, b)^{2}, \quad(a, b) \in \mathcal{U} .
$$

By a same method as Theorem 2.1, we have the following result for co-bi-Jordan homomorphisms.

Theorem 2.2. Every co-bi-Jordan homomorphism from commutative Banach algebra $\mathcal{U}$ into a semisimple commutative Banach algebra $\mathcal{D}$ is a co-bi-homomorphism.

We say that the Banach algebra $\mathcal{A}$ is weakly commutative if

$$
(a x)^{2}=a^{2} x^{2} \text { and } a x^{2} a=x^{2} a^{2},
$$

for all $a, x, \in \mathcal{A}$. Clearly, every commutative Banach algebra is weakly commutative, but in general, the converse is false. For example, let

$$
\mathcal{A}=\left\{\left[\begin{array}{ll}
a & b \\
0 & 0
\end{array}\right]: \quad a, b \in \mathbb{R}\right\} .
$$

Then it is obvious to check that with the usual matrix product for all $x, y \in \mathcal{A}$,

$$
(x y)^{2}=x^{2} y^{2} \quad \text { and } \quad x y^{2} x=y^{2} x^{2} .
$$

Thus, $\mathcal{A}$ is weakly commutative, but it is neither unital nor commutative.

Note that a unital Banach algebra is weakly commutative if and only if it is commutative.

Lemma 2.1. Let $\mathcal{U}$ be a weakly commutative Banach algebra, and $\varphi: \mathcal{U} \rightarrow \mathbb{C}$ be a bi-Jordan homomorphism. Then

$$
\varphi(a x, b y)=\varphi(a x, y b)=\varphi(x a, b y),
$$

for all $(a, b),(x, y) \in \mathcal{U}$.

Proof. By Lemma 1 of [13],

$$
\varphi\left(a^{2}, b y+y b\right)=2 \varphi(a, b) \varphi(a, y), \quad(a, b),(a, y) \in \mathcal{U} .
$$

Replacing $a$ by $a x$ in (2.6) we get

$$
\varphi\left((a x)^{2}, b y+y b\right)=2 \varphi(a x, b) \varphi(a x, y) .
$$

Replacing $b$ by by and $y$ by $y b$ in (2.7), gives

$$
\varphi\left((a x)^{2}, b y^{2} b+y b^{2} y\right)=2 \varphi(a x, b y) \varphi(a x, y b) .
$$


Since $\mathcal{U}$ is weakly commutative, by (2.8) we have

$$
\begin{aligned}
2 \varphi(a x, b y) \varphi(a x, y b) & =\varphi\left((a x)^{2}, b y^{2} b+y b^{2} y\right) \\
& =\varphi\left((a x)^{2}, y^{2} b^{2}+b^{2} y^{2}\right) \\
& =\varphi\left((a x)^{2}, b^{2} y^{2}\right)+\varphi\left((a x)^{2}, y^{2} b^{2}\right) \\
& =\varphi(a x, b y)^{2}+\varphi(a x, y b)^{2} .
\end{aligned}
$$

Thus,

$$
(\varphi(a x, b y)-\varphi(a x, y b))^{2}=0
$$

which proves that

$$
\varphi(a x, b y)=\varphi(a x, y b)
$$

for all $(a, b),(x, y) \in \mathcal{U}$. In a similar way, we can prove that $\varphi(a x, b y)=\varphi(x a, b y)$. This complete the proof.

The next result is the generalization of Theorem 2.1.

Theorem 2.3. Suppose that $\varphi$ is a bi-Jordan homomorphism from weakly commutative Banach algebra $\mathcal{U}$ into a semisimple commutative Banach algebra $\mathcal{D}$. Then $\varphi$ is a bi-homomorphism.

Proof. We first assume that $\mathcal{D}=\mathbb{C}$ and let $\varphi: \mathcal{U} \rightarrow \mathbb{C}$ be a bi-Jordan homomorphism. Then for all $(a, b) \in \mathcal{U}$,

$$
\varphi\left(a^{2}, b^{2}\right)=\varphi(a, b)^{2} .
$$

Replacing $a$ by $a+x$ and $b$ by $b+y$ in (2.9), gives

$$
\varphi(a x+x a, b y+y b)=2 \varphi(a, b) \varphi(x, y)+2 \varphi(a, y) \varphi(x, b),
$$

for all $(a, b),(x, y) \in \mathcal{U}$. It follows from (2.10) and Lemma 2.1 that

$$
\begin{aligned}
4 \varphi(a x, b y) & =\varphi(a x+x a, b y+y b) \\
& =2 \varphi(a, b) \varphi(x, y)+2 \varphi(a, y) \varphi(x, b) .
\end{aligned}
$$

Hence,

$$
2 \varphi(a x, b y)=\varphi(a, b) \varphi(x, y)+\varphi(a, y) \varphi(x, b),
$$

for all $(a, b),(x, y) \in \mathcal{U}$. Thus, the relation (2.4) in Theorem 2.1 holds. Now the rest of proof is similar to the proof of Theorem 2.1.

As a consequence of Theorem 2.3 we have the next result.

Corollary 2.1. Suppose that $\mathcal{U}$ is weakly commutative and $\varphi: \mathcal{U} \rightarrow \mathbb{C}$ satisfies

$$
|\varphi(a x, b y)-\varphi(a, b) \varphi(x, y)| \leq \delta(\|(a, b)\|+\|(x, y)\|),
$$

for all $(a, b),(x, y) \in \mathcal{U}$ and for some $\delta \geq 0$. Then $\varphi$ is a bi-homomorphism. 
Proof. Replacing $(x, y)$ by $(a, b)$ in $(2.11)$, gives

$$
\left|\varphi\left(a^{2}, b^{2}\right)-\varphi(a, b)^{2}\right| \leq 2 \delta(\|a\|+\|b\|),
$$

for all $(a, b) \in \mathcal{U}$. Take $a=2^{n} x$ and $b=2^{n} y$ in (2.12), then

$$
\left|\varphi\left(x^{2}, y^{2}\right)-\varphi(x, y)^{2}\right| \leq \frac{2^{n+1} \delta(\|x\|+\|y\|)}{2^{4 n}} \rightarrow 0
$$

as $n \rightarrow \infty$. Hence,

$$
\varphi\left(x^{2}, y^{2}\right)=\varphi(x, y)^{2}, \quad(x, y) \in \mathcal{U} .
$$

Therefore, $\varphi$ is a bi-Jordan and so it is a bi-homomorphism by Theorem 2.3.

Example 2.1. Let

$$
\mathcal{U}=\left\{\left(\left[\begin{array}{ll}
a & b \\
0 & 0
\end{array}\right],\left[\begin{array}{ll}
x & y \\
0 & 0
\end{array}\right]\right): \quad a, b, x, y \in \mathbb{R}\right\} .
$$

Then $\mathcal{U}$ is a weakly commutative Banach algebra, but it is not commutative. Hence by Theorem 2.3, each bi-Jordan homomorphism from $\mathcal{U}$ into a semisimple commutative Banach algebra $\mathcal{D}$ is a bi-homomorphism and via versa.

The commutativity of Banach algebra $\mathcal{D}$ in Theorem 2.3 is essential. For example, let

$$
\mathcal{A}=\left\{\left[\begin{array}{ll}
a & b \\
0 & 0
\end{array}\right]: \quad a, b \in \mathbb{R}\right\},
$$

as above and let $\mathcal{A}^{\sharp}$ be the unitization of $\mathcal{A}$ with the identity matrix as a unit. Set $\mathcal{U}=\mathcal{A} \times \mathcal{A}^{\sharp}$ and define $\varphi: \mathcal{U} \rightarrow \mathcal{A}$ by $\varphi(x, y)=x y$. Then for all $(x, y) \in \mathcal{U}$,

$$
\varphi\left(x^{2}, y^{2}\right)=\varphi(x, y)^{2} .
$$

Hence $\varphi$ is bi-Jordan homomorphism, but it is not bi-homomorphism. Because, let

$$
x=\left[\begin{array}{ll}
a & b \\
0 & 0
\end{array}\right], \quad y=\left[\begin{array}{ll}
c & d \\
0 & 0
\end{array}\right], \quad m=\left[\begin{array}{cc}
s & t \\
0 & 0
\end{array}\right] \quad \text { and } n=I,
$$

where $I$ is the identity matrix. Then $(x, y),(m, n) \in \mathcal{U}$, but

$$
\varphi(x m, y n) \neq \varphi(x, y) \varphi(m, n) .
$$

\section{Chracterization of 3-BI-Jordan Homomorhisms}

Clearly, the Banach algebra $\mathcal{U}$ is unital if and only if both $\mathcal{A}$ and $\mathcal{B}$ are unital. Without any confusion we denote by $e$, the unit element of both $\mathcal{A}$ and $\mathcal{B}$.

Lemma 3.1. Let $\mathcal{U}$ be a unital commutative Banach algebra, and $\varphi: \mathcal{U} \rightarrow \mathbb{C}$ be a 3-bi-Jordan homomorphism. Then for all $(a, b) \in \mathcal{U}$,

(a) $\varphi\left(a^{3}, b^{2}+b\right)=\varphi(a, b)^{2} \varphi(a, e)+\varphi(a, b) \varphi(a, e)^{2}$,

(b) $\varphi\left(a^{2}+a, b^{3}\right)=\varphi(a, b)^{2} \varphi(e, b)+\varphi(a, b) \varphi(e, b)^{2}$.

Proof. The proof is straightforward.

Lemma 3.2. By the hypotheses of above Lemma, for all $(a, b),(x, y) \in \mathcal{U}$, 
(a) $3 \varphi\left(a x^{2}, b\right)=\varphi(a, b) \varphi(x, e)^{2}+2 \varphi(a, e) \varphi(x, e) \varphi(x, b)$,

(b) $3 \varphi\left(a, b y^{2}\right)=\varphi(a, b) \varphi(e, y)^{2}+2 \varphi(e, b) \varphi(e, y) \varphi(a, y)$.

Proof. We prove (a), that the assertion (b) can be proved similarly. Let $\varphi: \mathcal{U} \rightarrow \mathbb{C}$ be a 3-bi-Jordan homomorphism. Then for all $(a, b) \in \mathcal{U}$,

$$
\varphi\left(a^{3}, b^{3}\right)=\varphi(a, b)^{3} .
$$

Replacing $b$ by $b+y$ in (3.1), gives

$$
\varphi\left(a^{3}, b^{2} y+b y^{2}\right)=\varphi(a, b)^{2} \varphi(a, y)+\varphi(a, b) \varphi(a, y)^{2},
$$

for all $(a, b),(a, y) \in \mathcal{U}$. Replacing $y$ by $-y$ in (3.2), we get

$$
\varphi\left(a^{3},-b^{2} y+b y^{2}\right)=-\varphi(a, b)^{2} \varphi(a, y)+\varphi(a, b) \varphi(a, y)^{2} .
$$

By (3.2) and (3.3) we have

$$
\varphi\left(a^{3}, b y^{2}\right)=\varphi(a, b) \varphi(a, y)^{2} .
$$

Replacing $y$ by $e$ in (3.4), gives

$$
\varphi\left(a^{3}, b\right)=\varphi(a, b) \varphi(a, e)^{2} .
$$

Replacing $a$ by $a+x$ in (3.5), to obtain

$$
3 \varphi\left(a x^{2}+a^{2} x, b\right)=I+J
$$

where

$$
I=\varphi(x, b) \varphi(a, e)^{2}+2 \varphi(a, b) \varphi(a, e) \varphi(x, e)
$$

and

$$
J=\varphi(a, b) \varphi(x, e)^{2}+2 \varphi(a, e) \varphi(x, b) \varphi(x, e) .
$$

Replacing $x$ by $-x$ in (3.6), we get

$$
3 \varphi\left(a x^{2}-a^{2} x, b\right)=-I+J
$$

By (3.6) and (3.7) we have

$$
3 \varphi\left(a x^{2}, b\right)=\varphi(a, b) \varphi(x, e)^{2}+2 \varphi(a, e) \varphi(x, b) \varphi(x, e),
$$

for all $(a, b),(x, e) \in \mathcal{U}$, as required.

Now we state and prove the main Theorem of this section.

Theorem 3.1. Suppose that $\varphi$ is a 3-bi-Jordan homomorphism from unital commutative Banach algebra $\mathcal{U}$ into $\mathbb{C}$. Then $\varphi$ is a 3-bi-homomorphism.

Proof. Let $\varphi: \mathcal{U} \rightarrow \mathbb{C}$ be a 3-bi-Jordan homomorphism. Then

$$
\varphi\left(a^{3}, b^{3}\right)=\varphi(a, b)^{3}, \quad(a, b) \in \mathcal{U} .
$$

Replacing both of $a$ and $b$ by $e$, gives $\varphi(e, e)=\varphi(e, e)^{3}$. Since $\varphi(e, e) \neq 0$, so $\varphi(e, e)=1$ or $\varphi(e, e)=-1$. We first assume that $\varphi(e, e)=1$. Replacing $a$ by $a+e$ and $b$ by $b+e$ in (3.8), and simplifies the result by Lemma 3.1, we get

$$
9 \varphi\left(a^{2}+a, b^{2}+b\right)=3\left\{\varphi(a, b)^{2}+\varphi(a, b)+P+Q+R+S\right\},
$$


where

$$
\begin{aligned}
P & =2 \varphi(a, b) \varphi(a, e)+\varphi(e, b) \varphi(a, e)^{2}, & Q & =2 \varphi(a, b) \varphi(e, b)+\varphi(a, e) \varphi(e, b)^{2}, \\
R & =2 \varphi(a, e) \varphi(e, b), & S & =2 \varphi(a, e) \varphi(a, b) \varphi(e, b) .
\end{aligned}
$$

It follows from preceding Lemma that for all $(a, b) \in \mathcal{U}$,

$$
P=3 \varphi\left(a^{2}, b\right), \quad Q=3 \varphi\left(a, b^{2}\right), \quad R=2 \varphi(a, b) \quad \text { and } \quad S=2 \varphi(a, b)^{2} .
$$

By (3.9) and (3.10) we obtain

$$
\varphi\left(a^{2}, b^{2}\right)=\varphi(a, b)^{2}
$$

for all $(a, b) \in \mathcal{U}$. Hence, $\varphi$ is bi-Jordan homomorphism and so it is bi-homomorphism by Theorem 2.1. Thus, $\varphi$ is 3 -bi-homomorphism.

Now suppose that $\varphi(e, e)=-1$. Then by a similar arqument we have

$$
\varphi\left(a^{2}, b^{2}\right)=-\varphi(a, b)^{2}, \quad(a, b) \in \mathcal{U} .
$$

Therefore by Theorem 2.2, $\varphi$ is co-bi-homomorphism. That is,

$$
\varphi(a x, b y)=-\varphi(a, b) \varphi(x, y),
$$

for all $(a, b),(x, y) \in \mathcal{U}$. Thus,

$$
\begin{aligned}
\varphi(\text { axu }, b y v) & =-\varphi(a, b)[\varphi(x u, y v)] \\
& =-\varphi(a, b)[-\varphi(x, y) \varphi(u, v)] \\
& =\varphi(a, b) \varphi(x, y) \varphi(u, v),
\end{aligned}
$$

for all $(a, b),(x, y),(u, v) \in \mathcal{U}$. So $\varphi$ is 3-bi-homomorphism, as claimed.

As a consequence of Theorem 3.1 we have the next result.

Corollary 3.1. Suppose that $\varphi$ is a 3-bi-Jordan homomorphism from unital commutative Banach algebra $\mathcal{U}$ into a semisimple commutative Banach algebra $\mathcal{D}$. Then $\varphi$ is a 3-bi-homomorphism.

In view of Theorem 1.1 and Theorem 2.1, the following question suggests itself: does Theorem 2.1 hold without commutativity of $\mathcal{U}$ ?

Acknowledgements. The author gratefully acknowledges the helpful comments of the anonymous referees.

This research was partially supported by the grant from Ayatollah Borujerdi University with No. 15664-137285. 


\section{REFERENCES}

[1] J. Bračič and M. S. Moslehian, On automatic continuity of 3-homomorphisms on Banach algebras, Bull. Malays. Math. Sci. Soc. 30(2) (2007), 195-200.

[2] M. Eshaghi Gordji, n-Jordan homomorphisms, Bull. Aust. Math. Soc. 80(1) (2009), 159-164.

[3] M. Eshaghi Gordji, T. Karimi and S. Kaboli Gharetapeh, Approximately n-Jordan homomorphisms on Banach algebras, J. Inequal. Appl. 2009 (2009), 1-8.

[4] E. Gselmann, On approximate n-Jordan homomorphisms, Ann. Math. Sil. 28 (2014), 47-58.

[5] Sh. Hejazian, M. Mirzavaziri and M. S. Moslehian, n-homomorphisms, Bull. Iranian Math. Soc. 31(1) (2005), 13-23.

[6] I. N. Herstein, Jordan homomorphisms, Trans. Amer. Math. Soc. 81 (1956), 331-341.

[7] Y. H. Lee, Stability of n-Jordan homomorphisms from a normed algebra to a Banach algebra, Abstr. Appl. Anal. 2013 (2013), 1-5.

[8] T. Miura, S.-E. Takahasi and G. Hirasawa, Hyers-Ulam-Rassias stability of Jordan homomorphisms on Banach algebras, J. Inequal. Appl. 2005 (2005), 435-441.

[9] W. Zelazko, A characterization of multiplicative linear functionals in complex Banach algebras, Studia Math. 30 (1968), 83-85.

[10] A. Zivari-Kazempour, A characterization of Jordan homomorphism on Banach algebras, Chinese J. Math. 2014 (2014), 1-3.

[11] A. Zivari-Kazempour, A characterization of 3-Jordan homomorphisms on Banach algebras, Bull. Aust. Math. Soc. 93(2) (2016), 301-306.

[12] A. Zivari-Kazempour, A characterization of Jordan and 5-Jordan homomorphisms between Banach algebras, Asian-Eur. J. Math. (2017) DOI 10.1142/S1793557118500213.

[13] A. Zivari-Kazempour, A characterization of bi-Jordan homomorphisms on Banach algebras, Int. J. Anal. 2017 (2017), 1-5.

${ }^{1}$ Department of Mathematics, Ayatollah Borujerdi University, BORUJERD, IRAN

Email address: zivari@abru.ac.ir, zivari6526@gmail.com 